\title{
THE MATHEMATICS OF FINANCE: PRICING DERIVATIVES
}

\author{
$\mathrm{BY}$ \\ STEPHEN A. ROSS \\ Franco Modigliani Professor of Finance and Economics, \\ Sloan School, Massachusetts Institute of Technology
}

Introduction. The recent Nobel prizes in economics awarded to financial economists confirmed the transition that finance has undergone in the past thirty years, moving from being what one wag called a taxonomy of anecdotes to being one of the more tangential and "softer" areas of economics to occupy center stage. Finance has come of age as a science in large part through the application of the work of Wiener and the other pioneers of the theory of stochastic processes. Diffusion processes have become part of the vocabulary of modern finance.

Accompanying this intellectual transformation has been a rare for the social sciences embracing of the practical significance - the engineering side - of finance. A recent article in Scientific American takes note of the hundreds of mathematicians and physicists who have moved to Wall Street, where they make use of quite advanced techniques in applied mathematics that lie at the heart of what has become a $\$ 50$ trillion industry. If one were to look for the new applications of mathematics one would be hard pressed to find an area where the impact has been more certain or more significant.

What I would like to do in this short paper is to give the reader a sense of what this is all about. What is happening on Wall Street and what are these mathematicians and physicists doing? What are the major research paradigms and what comes next?

The derivatives pricing problem-Call options. The central problem of modern finance is the valuation of assets and the streams of cash flows that they generate over time. These flows are generally uncertain and, as such, they are modelled as stochastic processes.

The best place to start is with a problem-indeed, the canonical problem of option pricing theory-that of valuing a call option on a share of stock. An option is a contract that gives its holder certain rights. A call option confers on the holder the right to purchase a share of stock on a certain date in the future and for a fixed price called the exercise or strike price. The terms of such contracts vary. American options can

Received May 29, 1997.

1991 Mathematics Subject Classification. Primary 90A12, 90A09.

I am grateful to Robert Merton and Andrew Lo for their helpful suggestions. 
be exercised at any time up to their expiration, whereas European options can only be exercised at a fixed date in the future. We will focus on European options.

An option is an example of a derivative. This term is used to emphasize that the option derives its value from the value of something else, in this case a share of stock, that underlies it and is primitive to the option.

Letting $S$ denote the price of a share, $K$ the strike price of the option, and $T$ its maturity, Figure 1 displays the familiar "hockey stick" value of this option at maturity, $T$, as a function of the terminal stock price at that time, $S_{T}$.

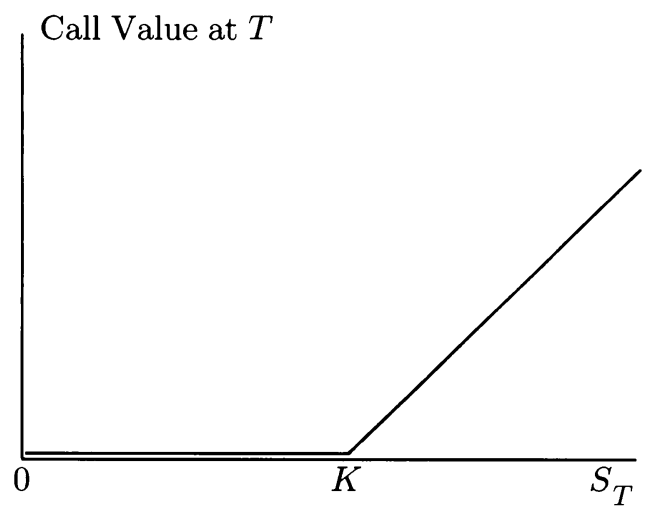

FIG. 1

If the value of the stock, $S_{T}$, is greater than the exercise price, $K$, then the option is said to be "in the money". The holder will now exercise it and purchase the stock for $K$. Since the stock is worth $S_{T}$, the resulting value of the option is the difference, $S_{T}-K$. (Not surprisingly, generally the market settles on this value for cash without invoking the cumbersome need to actually purchase the stock and transfer its ownership.) Alternatively, if $S_{T}$ falls short of $K$, then the option is "out of the money". In this case it is worthless and expires without being exercised since its holder would surely not want to exercise the right to purchase for $K$ something that is available in the market for the lower price of $S_{T}$. To summarize, at time $T$ the option has the value $\max \left[S_{T}-K, 0\right]$.

While there certainly are a great number of call options traded, they make up only a small part of the total volume of derivatives. Nevertheless, they are canonical not only because they were the first derivatives to be valued that had nonlinear payoffs, but also because they are the building blocks from which all derivatives can be valued. In fact, it is easy to see that these calls form a basis for the family of piecewise linear functions. Thus, if we can value call options, we can, in principle, value any function in their closure including but not limited to the continuous functions.

As an example, the stock itself is equivalent to a call with an exercise price of 0 . As a more interesting example, Fig. 2 displays a triangle function that has value in some range $[x, y]$ and peaks in the middle. This is called a butterfly spread and it is created by combining positive positions in calls with exercise prices of $x$ and $y$ with two negative positions in a call with an exercise price of $(x+y) / 2$. 


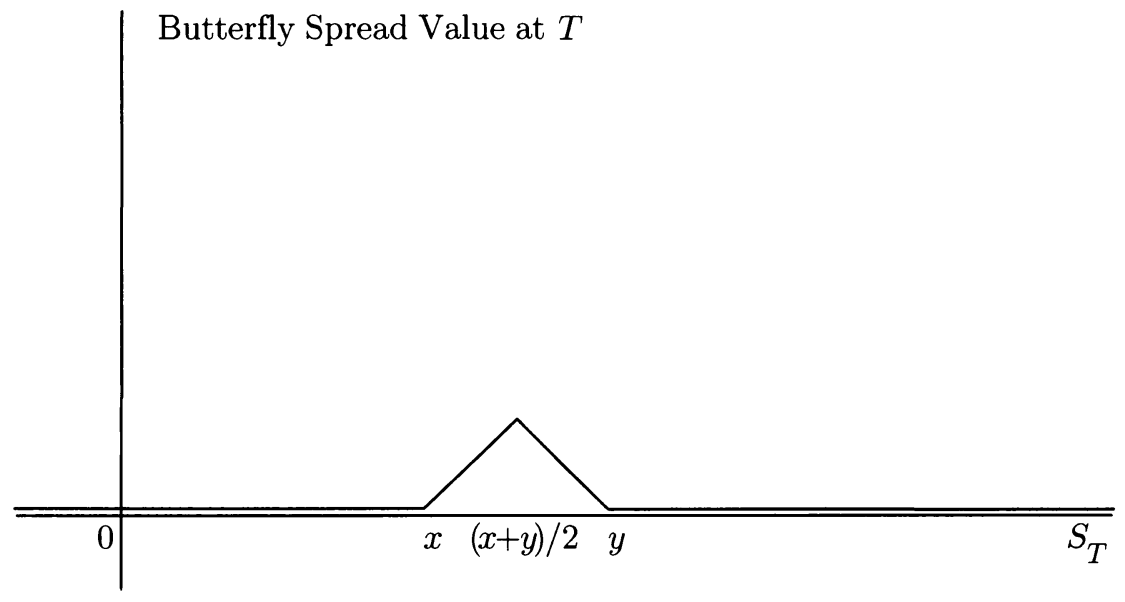

FIG. 2

1. The binomial approach. We know what the option is worth when it matures. Introducing some notation, we will let $C$ denote the value of the option, and let $t$ denote the time variable. At termination, $t=T$, the value of the call is given by

$$
C=\max \left[S_{T}-K, 0\right] .
$$

But, how do we determine the value $C_{t}$ for $t<T$ ?

While it is possible to find some process-free bounds for $C_{t}$-for example, we know a priori that $C(t)<S(t)$-in general we expect that $C_{t}$ will depend upon the movement of the stock price over time, i.e., the stochastic process that describes the motion of $S_{t}$.

Moreover, since at termination the option value depends on the stock price, it follows that at time $t<T$ its value will generally depend on any variables that are stochastically related to the distribution of the stock value at time $T$. The simplest interesting process is the binomial process in which the stock price moves on a discrete lattice going either up or down by a proportionate discrete amount at each node of the lattice. This process is depicted in Fig. 3.

The tree in Fig. 3 displays the evolution of the information process that underlies the model. Each limb represents a particular resolution and refinement of that uncertainty. With obvious notation, suppose the process is at node $(t, S)$. At time $t+1$ the stock price has to change to either the up value, $a S$, or the down value $b S$, where $b<a$. This proportionate change tree is used-rather than an absolute tree with fixed changes - to avoid the possibility of the stock's price becoming negative.

Writing the value of the option as a function of time and the stock value, $C(t, S)$, it is clear that, like the stock, $C(t, S)$ will follow the binomial process illustrated in Fig. 4 (see p. 699). In addition to the stock and the option, we will also assume that a risk free bond is traded and, for simplicity, we will let it carry a fixed interest rate of $r$ in each period. In other words, 1 dollar lent out from period $t$ to $t+1$ will be worth $1+r$ dollars at time $t+1$. 


\section{Binomial Tree}

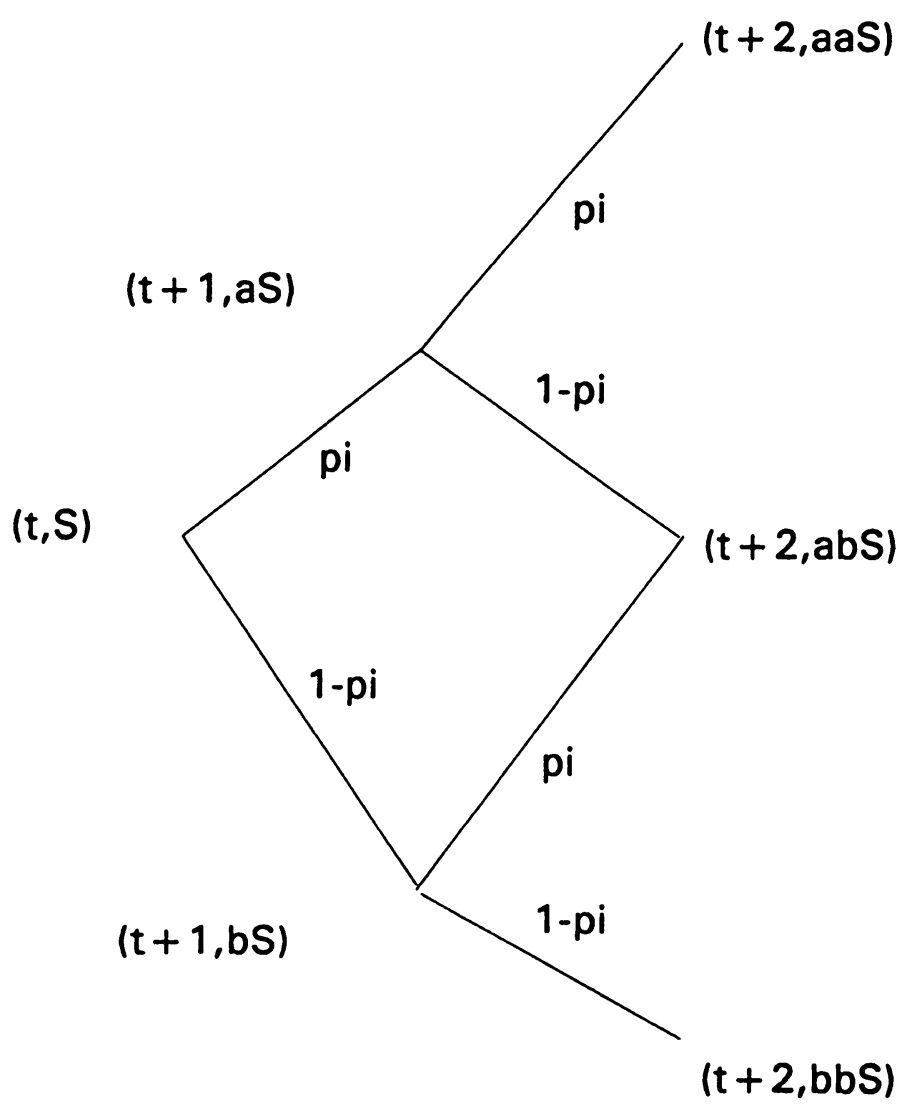

FIG. 3

We will assume that the bond, the stock, and the option all trade in liquid markets with no frictions or costs. To solve the problem of determining the value of the option, typically economists would make some further assumptions necessary to create a model of demand and supply, the solution to which would be the equilibrium price of the option. Such a model would make use of investors' preferences towards risk to construct a demand curve for the option. Presumably, for example, since an option receives a zero payoff if it matures out of the money, i.e., if $S_{T}<K$, options are riskier than stocks and, a priori, we would expect that economies filled with more risk-averse investors would bid down the prices of options compared to economies with more risk-tolerant investors. Fortunately, for both the theory and the practice of option pricing, this is not the path that we will follow. There is a detour to a quicker and far more elegant solution. 


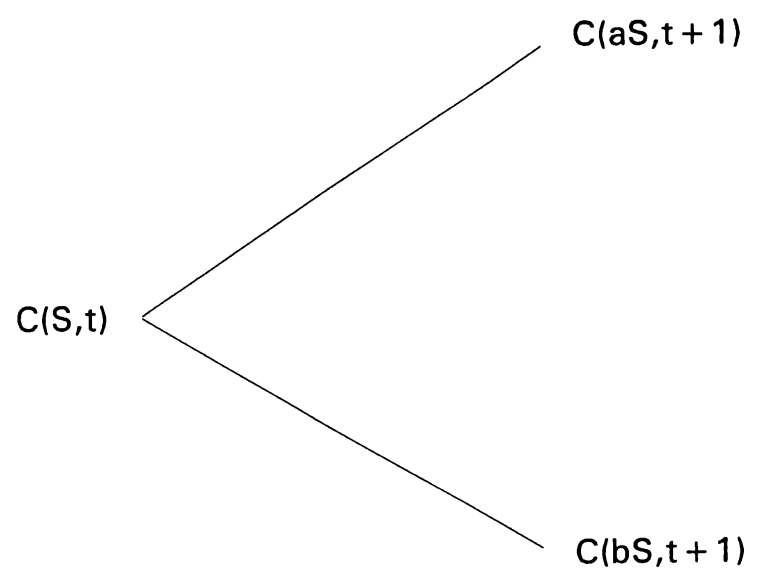

FIG. 4

An investor in this world has a variety of possible decisions open, including borrowing, lending, or dividing investments in some proportion among all three of these possibilities. Begin by considering the choice between stocks and bonds. An investment of $\$ 1$ in the stock will either be worth $b$ or $a$ at time $t+1$. An investment in the bond will be worth $1+r$ in either case. Clearly, if $b>1+r$, then the stock dominates the bond. What would an investor do in this case? Well this is a wonderful world to live in -in fact, it is too wonderful. Not merely would investors put their wealth in stocks rather than bonds, there is no reason to stop at that. Borrowing one dollar at the interest rate $r$ and investing it in the stock will reap a minimum value of $b$ at time $t+1$. Since $b$ exceeds $1+r$, a positive amount $b-(1+r)$ would be left to pocket as profit. Why stop there? If $\$ 1$ will do, why not $\$ 1$ million or $\$ 1$ billion or more? Similarly, if $b=1+r$, then borrowing to buy the stock is sure to never lose and to return a positive gain on the up (state $a$ ) leg. This is a truly "no lose" situation-a bit like the arguments of those who would have you put all of your investments in stocks because "stocks always beat bonds".

Such a situation is called an arbitrage possibility, and, unfortunately, neither the real world nor any economic model of equilibrium allows such things. Very quickly any temporary appearance of an arbitrage will be closed down by the funds it attracts. There is a joke told in introductory finance classes that captures the matter quite well. The student and the professor are walking down the hall and they see a $\$ 20$ bill on the ground. The student bends down to pick it up and the professor says, "Don't bother, if it was really there, someone else would have picked it up already."

A similar situation in reverse arises if $1+r \geq a$, in which case the bond dominates the stock and there would be no demand for the stock. Hence, the absence of arbitrage, or "no arbitrage" for short implies that we must have $b<1+r<a$.

Of course, investors are not required to put all of their portfolio into either the stock or the bond; they can divide their wealth between the two. Suppose that at node $(S, t)$ the investor forms a portfolio with $x$ invested in the stock and $1-x$ loaned out at the rate $r$. The gross return on the portfolio is the linear combination of the return on the 
stock, $a$ or $b$, and that from the loan, $1+r$. In state $a$, the portfolio returns

$$
x a+(1-x)(1+r)
$$

and in state $b$ it returns

$$
x b+(1-x)(1+r) .
$$

By comparison, $\$ 1$ invested in the option purchases $1 / C(S, t)$ options at time $t$. In state $a$, at time $t+1$, each option has the value $C(a S, t+1)$ producing a total return of

$$
C(a S, t+1) / C(S, t)
$$

and in state $b$ the total return is

$$
C(b S, t+1) / C(S, t) .
$$

Since we are free to choose any investment proportion, $x$, we wish, suppose that we choose $x$ in such a way as to make the portfolio and the option have the same return in state $b$ :

$$
x b+(1-x)(1+r)=C(b S, t+1) / C(S, t),
$$

which implies that

$$
x=\frac{(1+r)-C(b S, t+1) / C(S, t)}{(1+r)-b} .
$$

But, if the portfolio has the same return as the option in state $b$, then what can we say about its return in state $a$ ? Recall the argument comparing the returns on a unit investment in the stock with lending and borrowing. The same line of reasoning now applies to the comparison between the portfolio we have just constructed and the option. If the portfolio beats the option in state $a$, then why would anyone buy the option? In fact, rather than holding the option, investors would issue (sell) options and use the proceeds to buy the portfolio earning an arbitrage profit.

Conversely, suppose that the option beats the portfolio in state $a$. In this case we would reverse the investment in the portfolio, i.e., borrow $(1-x)$ and purchase $-x$ dollars of stock, which is the same as "shorting" $x$ worth of stock. This would generate $\$ 1$ in cash, which could be invested in the option. Paying back the loans on this reversed portfolio would thereby produce a zero net return in state $b$ and a positive return in state $a$. If shorting a portfolio seems mysterious there is an alternative way to make the same point. We can work through the algebra to show that if the option's returns in state $a$ exceed the portfolio's, then a new portfolio composed of lending and investing in the option could be constructed that would dominate the stock. Hence, investors would all prefer this portfolio to holding the stock, which would also be an arbitrage opportunity.

To prevent arbitrage, then, we are forced to require that if the portfolio has the same return as the option in state $b$ then it must also be the same in state $a$,

$$
x a+(1-x)(1+r)=C(a S, t+1) / C(S, t) .
$$

Figure 5 displays this result geometrically. Clearly, all three securities must lie on a common line to prevent arbitrage. 


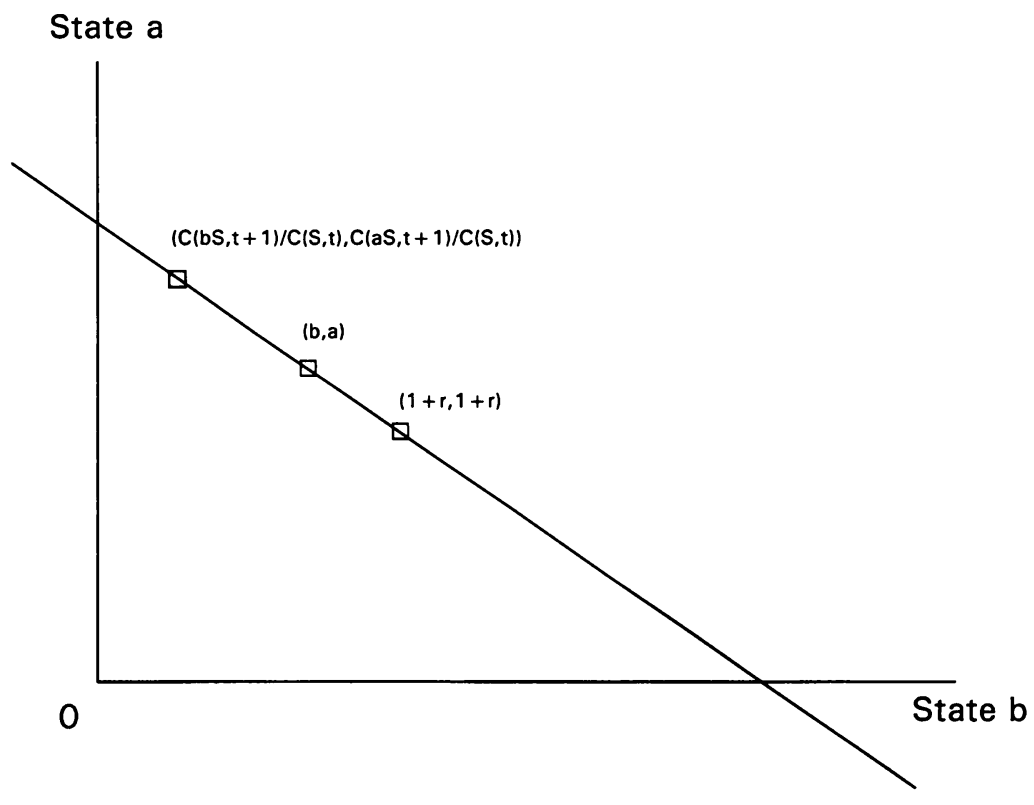

FIG. 5

Putting these results together produces a difference equation that the option value must satisfy,

$$
\pi^{*} C(a S, t+1)+\left(1-\pi^{*}\right) C(b S, t+1)-(1+r) C(S, t)=0
$$

where

$$
\pi^{*}=\frac{(1+r)-b}{a-b} .
$$

It is easy to solve this equation by working backward recursively from the boundary value,

$$
\begin{gathered}
C(S, T)=\max (S-K, 0) \\
C(S, t)=\frac{1}{(1+r)^{T-t}} \sum \pi^{* j}\left(1-\pi^{*}\right)^{(T-t-j)}\left(S a^{j} b^{(T-t-j)}-K\right)^{+},
\end{gathered}
$$

where the superscript + indicates the positive part.

There is something unexpected and remarkable about this result. Notice that the probability of an up jump, $\pi$, does not appear in the difference equation and, therefore, it does not show up in the solution. Consider what this means. Take two stocks that both follow binomial processes and that are not perfectly correlated. Further, suppose that the stocks differ only in that one has a much higher probability of an up jump than does the other. If our analysis is to be believed, then when the stock prices of each are equal the two option values will be equal! How can this be? How can the value of an option on a stock be independent of the probability that the stock will go up?

The resolution of this paradox lies in yet another paradox. If it is all that clear that the option on the process with the higher probability of an up jump should be more valuable, 
then why is not the stock itself more valuable? After all, the stock is an option with a zero strike price. By fixing the price of the two stocks to be equal we are implicitly saying that the market values the future development of the stocks equally. In equilibrium the prices already reflect the fact that one has a better chance of rising than the other; hence, there must be some implicit rationale for valuing them equally. This same implicit force is what equates the two option values.

The diffusion model-Black-Scholes. While the binomial model is the simplest derivation of the theory of option pricing, historically, it was preceded by a derivation in the mathematically more complex setting of a continuous-time model of uncertainty.

Letting the binomial process converge to a log-normal version of a continuous-time diffusion process yields

$$
d S=\mu S d t+\sigma S d z
$$

where $\mu$ is the local drift and $\sigma$ is the local speed or standard deviation of the continuoustime process.

Similarly, the difference equation becomes a partial differential equation,

$$
\frac{1}{2} \sigma^{2} S^{2} C_{S S}+r S C_{S}-r C=-C_{t}
$$

with a solution - subject to appropriate boundary conditions - that is the limit of the discrete binomial solution,

$$
C(S, t)=S N\left(d_{1}\right)-e^{-r(T-t)} N\left(d_{2}\right),
$$

where $N$ denotes the cumulative normal distribution function, and

$$
\begin{gathered}
d_{1}=\frac{\ln (S / K)+\left(r+\frac{1}{2} \sigma^{2}\right)(T-t)}{\sigma(T-t)^{1 / 2}}, \\
d_{2}=d_{1}-\sigma(T-t)^{1 / 2} .
\end{gathered}
$$

This is the famous Black-Scholes differential equation first discovered by Fischer Black and Myron Scholes [1973] and developed and studied by Bob Merton [1973] nearly 25 years ago while they were all new faculty members at MIT. Like the binomial model first analyzed by Cox, Ross, and Rubinstein [1979], the continuous-time model has a similar paradox; notice that the mean return, $\mu$, has no impact on the option value.

In both of these models the paradox provides a clue to the solution. Since the solution does not depend on deep parameters, and follows from arbitrage alone, it follows that the same solution would prevail in any economic equilibrium. In particular, the solution is the same as would occur in a world where investors were risk neutral and cared only about expectations. This is called the risk neutral or martingale approach and it is equivalent to valuing the option as the expected discounted terminal value using the risk-adjusted measure. Referring back to the binomial model, the constant, $\pi^{*}$, is a probability, which is to say, that it is positive and less than one. It is, in fact, the very risk-adjusted probability that we are talking about and an examination of the solution to the binomial model reveals that it is simply the discounted expected value of the option at maturity using $\pi^{*}$ as the probability of an up jump. In continuous time, the diffusion process with drift $r d t$ is the proper risk-adjusted measure and the solution to the Black-Scholes 
equation is the discounted expected value of the option at maturity using the probability for the stock price generated by this adjusted process (see Cox and Ross [1976]).

This analysis - called the risk neutral or martingale analysis - has become the workhorse for derivative pricing and has led to myriad innovations in the field. It has also sparked the interest of probabilists and all manner of mathematicians.

The absence of the probabilities in the binomial model and the drift in the continuoustime model is of immense practical significance. Indeed, were this not so, the theory would be robbed of much of its importance. Both the mean and the jump probabilities are very difficult to measure accurately in practice. For a stock with an annual standard deviation for its returns of $40 \%$ and a mean of $10 \%$ it would take 64 years of observing returns before we could be assured statistically with probability of .95 that the mean was actually even positive.

By contrast, we can, in principle, measure the speed, $\sigma$, of the process with arbitrary precision by sampling more densely within in any finite interval without any need to extend the time period of observation. This eliminates the need to reconcile any divergent views that investors might have about the future prospects for the stock or primitive assets. It also means that the theory can accommodate quite complex stochastic drifts, $\mu$.

\section{Solved and unsolved problems.}

Two "solved" problems: The term structure and integral approaches to optimization. Research in derivatives pricing has exploded at such a pace that there are now numerous new journals struggling to keep up, and in a short space it is impossible to do more than select some examples of what has been accomplished and what looms as important and unsolved.

One area that has attracted an enormous research focus is the theory of the term structure of interest rates. The term structure of interest rates, by which I mean the time profile of interest rates on bonds of different maturities, say, one year, two year, and so forth, can now be modelled in a rigorous fashion. By breaking the movement of the term structure into independent factors or Markov state variables and treating bonds themselves as derivatives whose value is conditional on the movement of interest rates, this theory has proven immensely successful. Figure 6 illustrates a common breakdown of the movement of the term structure into three basis factors each of which moves according to some specified stochastic process. At a practical level this approach has allowed us to price and create all manner of interest-dependent securities from mortgages to convertible bonds.

Another area of great success has been the development of integral or complete market approaches to optimization. This is sometimes called optimization without dynamic programming. We can do little more than outline the approach here. Consider the problem of maximizing the expected value of some integral of a function of a diffusion at some future date, $T$ :

$$
\max \int U(x(T)) d F_{x(T)}
$$




\section{Basic Factors in the Term Structure}

\section{Shift}

Interest Rate

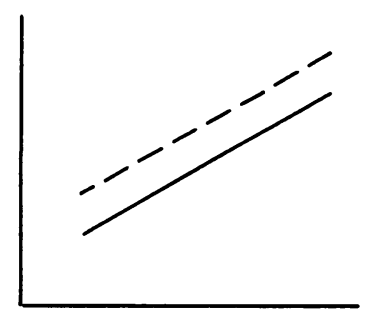

Bond Maturity
Tilt

Interest Rate

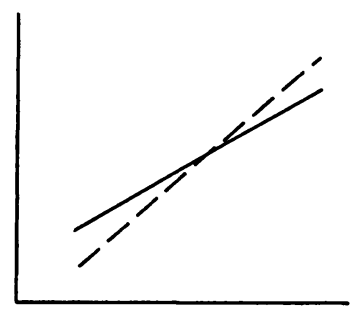

Bond Maturity
Flex

Interest Rate

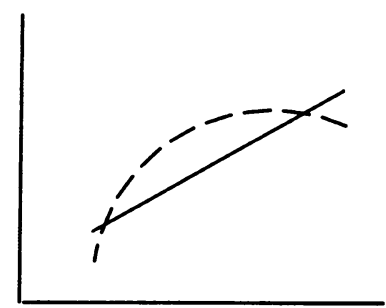

Bond Maturity

FIG. 6

where the maximization is over some control variables such as the proportion invested in stocks or bonds at time $t \in[0, T]$ and $s$ denotes the state of nature at time $T$, e.g., in a Markov diffusion, the value of the stock price $S(T)$ at time $T$, or, more generally, the entire path of the underlying state variables up to $T$. Typically we would attack this by dynamic programming techniques. Here is another approach. From our previous work we know that we can, in effect, purchase any pattern of payments $x(T)$ as a function of the disconnected martingale probabilities, $\pi^{*}(s, T)$, that describe the risk-adjusted probabilities of being in a certain state $s$ at time $T$. The only constraint is that we not exceed our initial wealth, $w_{0}$,

$$
\int x(s, T) d \pi^{*}(s, T)=w_{0} .
$$

But this implies that the optimal solution is an option,

$$
x(s, T)=U^{\prime-1}\left(\lambda \frac{d \pi^{*}}{d F}\right)
$$

where the Radon-Nikodym derivative is just a function of the terminal state, say the stock price, $S(T)$, and $\lambda$ is a Lagrange multiplier.

Now we have described the solution to our problem as an option whose terminal value $x(s, T)=f(S(T))$, and we can use the pricing theory developed earlier to fully characterize the solution. This approach works in quite complex problems where, for example, $U(\cdot)$ is messy and the differential equations of dynamic programming are highly nonlinear.

Two unsolved problems: The American put and other free boundary problems and spanning by options. The option problem we solved above was a European call option. A European call can only be exercised at maturity. By contrast, an American call can be exercised at any time up to and including the maturity date. Sometimes this distinction 
does not matter; if the stock pays no dividend then the two are identical because it would never pay to prematurely exercise the call. If the stock pays a dividend, though, early exercise may be optimal. Similarly, an American put will always be exercised early if the stock price is low enough.

A put gives the holder the right to sell the stock for a fixed amount or exercise price, $K$. Clearly, if the stock becomes nearly worthless at some time $t<T$, the time the put expires, it will pay to exercise the put, collect $K-S(t)$, and earn interest on this amount rather than wait till $T$. In both the case of the call on the dividend-paying stock and the put on a stock, whether dividend-paying or not, there is an optimum boundary at which to exercise. This optimal choice of the boundary is unsolved except in a handful of special cases and these are classic examples of what are called free boundary problems in the theory of differential equations. While numerical analyses are available, a full treatment is not and very little is known about the general theory of such problems.

Understanding these will lead to a better understanding of an equally deep and as yet unresolved issue. We do not know how rich is the space of all contracts that can be spanned in some appropriate sense by derivative securities. A contract is really just a bundle of contingent statements and payments. We are working to develop a theory that would allow us to break these contracts into their component parts and the roles played, for example, by American versus European derivatives as bases for such a construction is not yet resolved. In a sense, this is the financial economists' version of the biologists' effort to map the structure of DNA and one can only begin to imagine what we would learn from it.

Conclusion. The Black-Scholes differential equation is the backward equation for this process, and this particular equation is a transformation of the heat equation. The call option valuation problem is a boundary value problem and little more needs to be said about why Wall Street wants to hire physicists and mathematicians. All of the contracts currently traded are variations of the two simple models presented today. Since most are too complex for closed form solutions, they are solved using risk neutral versions of the binomial model or related discrete time models. (In fact, the solutions are Feynman-Kac integrals, which are generally solved by numerical or Monte-Carlo techniques.)

How well does this theory work? I once worked on a consulting project for a company that wanted a statistical estimate of the demand for Scotch whiskey in Massachusetts. Armed with the latest techniques in econometrics and statistics, I gathered the data, ran my regressions and estimated the demand curve. Try as I could, I could not get that curve to slope downward. The higher the price of Scotch, the more people drank. This was a disillusioning but by no means unusual exercise in empirical economics. By contrast, if you open up the newspaper and look at the option tables, the formulas in this paper come within small change of the quotes. And, the signs are all correct!

This close interplay between data and theory is characteristic of science and it characterizes modern finance. Equally characteristic is the exciting work yet to be done. 


\section{BIBLIOGRAPHY}

[1] F. Black and M. Scholes, The pricing of options and corporate liabilities, Journal of Political Economy 81(3), May-June, 637-654 (1973)

[2] J. C. Cox and S. A. Ross, The valuation of options for alternative stochastic processes, Journal of Financial Economics 3(1-2), January-March, 145-166 (1976)

[3] J. C. Cox, S. A. Ross, and M. Rubinstein, Option pricing: A simplified approach, Journal of Financial Economics 7(3), September, 229-263 (1979)

[4] Jon Ingersoll, Theory of Financial Decision Making, Rowman and Littlefield, 1987

[5] R. C. Merton, Theory of rational option pricing, Bell Journal of Economics and Management Science 4(1), Spring, 141-183 (1973) 